\title{
Risikofaktor Malnutrition bei Tumorpatienten
}

\begin{abstract}
Malnutrition ist bei onkologischen Patienten komplikationsreich und erhöht die Mortalität. In einer Beobachtungsstudie wurde das Risiko für eine Mangelernährung bei längeren Klinikaufenthalten untersucht.
\end{abstract}

Z

ahlreiche Komplikationen ergeben sich für onkologische Patienten, wenn die Ernährung nicht mehr stimmt: von Komorbiditäten und längerem Klinikaufenthalt bis zur Abnahme von Überlebensaussichten und Lebensqualität. Die Ursachen für Malnutrition sind vielfältig - von Stoffwechselveränderungen infolge der Neoplasie bis zu ungenügender Nahrungsaufnahme (wegen $\mathrm{Mu}-$ kositis, Diarrhö, Erbrechen, Schluckbeschwerden u.a.). Kachexie betrifft $80 \%$ der Patienten mit fortgeschrittener Krebserkrankung und ist für knapp ein Drittel aller krebsbedingten Todesfälle mitverantwortlich. In einer multizentrischen Beobachtungsstudie wurde die Prävalenz von Risikofaktoren bei 401 onkologischen Patienten untersucht. Der Ernährungszustand wurde mithilfe des Nutrition Risk Screening ${ }^{\circ}-2002$ (NRS ${ }^{\oplus}$-2002) innerhalb der ersten 48 Stunden nach Aufnahme sowie bei Entlassung ermittelt. Am häufigsten waren

Tab. 1: Kosten und Dauer der Hospitalisierung je nach Ernährungsstatus

Ernährungsstatus des Patienten bei

Aufnahme

Entlassung

NRS $<3$

NRS $\geq 3$

NRS $<3$

NRS $\geq 3$

Länge des Krankenhausaufenthaltes

9,3 Tage

11,7 Tage

8,6 Tage

12,1 Tage

Kosten des Krankenhausaufenthaltes

7.033 US-\$

7.855 US-\$

6.652 US-\$

8.596 US-\$

NRS $=$ Nutrition Risk Screening ${ }^{\circ}$-2002; modifiziert nach Planas et al.

\section{Transdermale Emesisprophylaxe, eine Option}

\section{Eine wichtige Säule der Prophylaxe von Übelkeit und Erbrechen bei emeto- gener Chemotherapie sind $5-\mathrm{HT}_{3}$-Rezeptorantagonisten $\left(5-\mathrm{HT}_{3}-\mathrm{RA}\right)$, darunter Granisetron. Welchen Vorteil bietet die transdermale Galenik des Wirkstoffs?}

n einer multizentrischen, randomisierten Phase-IV-Studie mit Crossover wurde das neue „Granisetron transdermal delivery sytem" (GTDS) mit Palonosetron verglichen. Das GTDS gibt den Wirkstoff kontinuierlich über 7 Tage ab.

Insgesamt wurden 196 Patienten, die sich einer moderat emetogenen Chemotherapie (FOLFIRI oder FOLFOX) unterzogen, randomisiert: In der GP-Gruppe erfolgte die Emesisprophylaxe im 1. Zyklus mit dem GTDS-Pflaster, im 2. mit Palonosetron (einmalig $0,25 \mathrm{mg}$ intravenös an Tag 1), in der PG-Gruppe war das Vorgehen umgekehrt. Primärer Endpunkt war der Anteil an Zyklen mit komplettem Ansprechen auf die Prophylaxe (kein Erbrechen, keine Rescue-Medikation) während der akuten Phase (024 Stunden nach Chemotherapie).

In der Analyse wurden 333 Chemotherapie-Zyklen berücksichtigt, davon 165 mit GTDS- und 168 mit PalonosetronProphylaxe. Ein komplettes Ansprechen in der akuten Phase wurde in $75,2 \%$ der Zyklen mit GTDS- und 79,8\% derer mit Palonosetron-Prophylaxe erreicht. Auch am Ende des 1. bzw. 2. Zyklus zeigte sich hinsichtlich des kompletten Anspre-
Tumoren des unteren Gastrointestinaltrakts (19,5\%), hämatologische Neoplasien (16\%), Kopf-Hals-Tumoren (8,5\%), Tumoren von Respirationstrakt $(8,0 \%)$, oberem Gastrointestinaltrakt (4,2\%) und von Bauchspeicheldrüse, Leber oder Gallengängen (4,2\%).

Etwa ein Drittel der Patienten (33,9\%) hatte bei Einweisung ein Risiko für Mangelernährung (NRS ${ }^{\circ}-2002 \geq 3$ ), bei Entlassung 36,4\%. Die betroffenen Patienten waren im Durchschnitt älter, Körpergewicht und Body-Mass-Index waren geringer, ebenso Arm- und Wadenumfang. Zudem waren die Serumalbumin-Spiegel niedriger. Die Gesundheitskosten waren bei Patienten mit Ernährungsrisiken höher und die mittlere Dauer des Klinikaufenthalts länger $(12,1$ Tage) als bei wohlernährten Patienten (8,6 Tage) (Tab. 1).

Fazit: Hospitalisierte, v.a. ältere Krebspatienten haben ein hohes Risiko für Malnutrition, müssen länger in der Klinik bleiben und verursachen höhere Kosten. Außerdem verschlechtert sich ihr Ernährungsstatus während des Klinikaufenthalts. Kathrin von Kieseritzky

Planas M et al. Prevalence of hospital malnutrition in cancer patients: a sub-analysis of the PREDyCES ${ }^{\circledR}$ study. Support Care Cancer. 2016; 24(1):429-35.

chens während der akuten Phase kein Unterschied zwischen den Gruppen ( $\mathrm{p}=0,405$ bzw. 0,074$)$.

Die Zufriedenheit der Patienten, gemessen anhand des FLI-E (Functional Living Index-Emesis) war in der GPGruppe in den Zyklen mit GTDS-Prophylaxe größer (FLI-E-Score median $1.549,5$ vs. $1.670,0)$.

Fazit: Transdermales Granisetron ist in der Prophylaxe von Übelkeit und Erbrechen bei moderat emetogener Chemotherapie eine gute Alternative $\mathrm{zu} \mathrm{Pa}-$ lonosetron. Kathrin von Kieseritzky

Seol YM et al. Transdermal granisetron versus palonosetron for prevention of chemotherapy-induced nausea and vomiting following moderately emetogenic chemotherapy: a multicenter, randomized, open-label, cross-over, active-controlled, and phase IV study. Support Care Cancer. 2016;24(2):945-52. 